\title{
Introduction: Menstruation as Structural
}

\author{
Inga T. Winkler
}

To recognize menstruation as structural, we must tune into the very political dimensions that undergird our institutions, laws, policies, budgets, guidelines, taxation, programs, and data collection. Historically, decision-makers have paid limited attention to menstruation-either due to oversight and neglect or due to deliberate exclusion. Yet, the last decade has seen enormous developments; at various levels, menstruation is rising to the level of global awareness. This might be what most distinguishes the current state of the menstrual movement from its past. Menstruation is gaining traction.

Examples of this shift abound. We see an emerging field of 'menstrual hygiene management' as development practice. Policy advocacy is burgeoning, for example addressing 'period poverty' in the United Kingdom and 'menstrual equity' in the United States. Efforts to improve menstrual hygiene and health range from innovating and distributing products, building girl-friendly facilities in schools, raising awareness through broad-based campaigns such as establishing May 28 as Menstrual Hygiene Day, campaigning to lift the 'tampon tax,' developing national menstrual health policies in India, Kenya, Senegal, and many other countries, to embedding menstrual health in the Sustainable Development Goals.

Against this background, this section offers an overview and early assessment of these developments at various levels including those driven by practitioners, policy-makers, activists, and civil society actors. It seeks to capture these trends and initiatives through a combination of practice-based and research-based chapters that bring together different perspectives, voices, and experiences. This diversity is essential to engage different types of emerging knowledge in this field and to combine practical experience with critical reflection.

The section starts with a reflection by Catarina de Albuquerque and Virginia Roaf on the origins of raising awareness on menstrual hygiene in the 
context of human rights. The account demonstrates that the water, sanitation and hygiene (WASH) sector has played a critical role in the early development of these efforts as it provided an entry point. However, they also acknowledge that there are obvious, but underutilized linkages with other fields including health, education, work, sexual and reproductive health and rights, culture, religion, among many others.

While these global developments have played a vital role in raising the profile of menstruation, governments and other actors at the national level have been instrumental in developing policies, programs, tax reforms, and advocacy campaigns. Archana Patkar traces the development of such efforts in multiple countries, exploring the pathways, challenges, and successes of embedding menstrual health in formalized national agendas. Swatija Manorama and Radhika Desai complement this policy analysis by taking a critical look at the gaps in Indian health policies, while Rockaya Aidara and Mbarou Gassama Mbaye offer insights into a menstrual hygiene management program in West Africa. Jennifer Weiss-Wolf offers an account of her involvement with the 'menstrual equity' movement in the United States, including her reflections on the elimination of the 'tampon tax' as a gateway for activism. The strongest testament to the deeply political nature of menstruation is revealed by Stella Nyanzi's essay in which she details her bold efforts to hold the Ugandan government accountable for its promise to provide menstrual products to girls leading to her imprisonment for her critique of the Ugandan President, Yoweri Museveni.

Another key policy debate is about menstrual leave, which Rachel Levitt and Jessica Barnack-Tavlaris address in their chapter. This debate, possibly more than any other, shows that policies and other structural changes must not be developed in isolation, but accompanied by sociocultural resistance to menstrual stigma and gendered stereotypes. Introducing a menstrual leave policy in a particular context that is not yet equipped for it might have unintended negative repercussions for menstruators. On the other hand, the introduction of menstrual leave policies and other legislative, policy, and judicial developments serve to normalize menstruation and, in that way, can be very powerful.

In the subsequent two chapters, Libbet Loughnan et al. turn to exploring menstruation specifically in the context of the Sustainable Development Goals and the monitoring frameworks they provide, and Siri Tellier et al. address humanitarian responses that point to the challenges that arise in the immediate aftermath of disasters as well as long-term protracted crises.

While menstrual hygiene and health programming is a rapidly developing and dynamic field, this section seeks to provide an overview of the current state of knowledge, which also prompts us to ask 'where do we go from here?'. Venkrataman Chandra-Mouli and Sheila Vipul Patel map the knowledge of menarche and menstrual health among adolescent girls and generally find low levels of knowledge in many low- and middle-income countries, and the same holds true for many girls living in the Global 
North. Just as importantly, the section also acknowledges and draws attention to the fact that there is a lot we still do not know, while program and policy responses are moving at a rapid pace. Julie Hennegan provides a critical analysis of the evidence for menstrual health interventions in low- and middle-income countries, discussing the approaches which have been trialed to date and highlighting key gaps and new potential pathways. The "Transnational Engagements" edited by Victoria Miller and Inga T. Winkler on the emergence of the field of menstrual health and hygiene conclude this section and similarly raise important questions about the status, development, and future directions of the field, which warrant further exploration. The rapid momentum around menstruation as focus of intervention brings significant opportunities, but it also poses risks.

First, several of the chapters highlight the centrality of WASH in spiraling attention to menstrual hygiene. WASH is a largely technical sector focused on facilities and infrastructure. Moreover, many of the initiatives described in this section are focused on products: their distribution, use, regulation, safety, and taxation. While these initiatives can make valuable contributions (and surely most menstruators assert the need for something to bleed on or into), we observe a narrow focus on the provision of menstrual products as 'quick fixes,' losing sight of structural injustices, which can be addressed through the lens of menstruation. This narrowed focus risks obscuring broader questions of gender justice and body politics which underlie sociocultural perceptions of menstruation. Too few programs tackle the embarrassment and shame associated with menstruation at a deeper level and instead often reiterate (sometimes inadvertently) that menstruation is best kept concealed. We often hear that, for instance, initiatives in the WASH sector or the elimination of the 'tampon tax' can provide entry points for tackling the broader, structural challenges. It is time to honor that promise and to move from the entrance into the deeper structure. Only then will we be able to normalize menstruation.

Second, there is a risk of rushing toward poorly conceived policies that are developed on the basis of sensationalized accounts instead of reliable evidence. The widely cited claim that one in ten 'African' girls allegedly miss school because of menstruation, is one recent example of a claim without backing. The contributions in this section caution us to develop a solid evidence base for policy and program development. While many (in particular qualitative) studies detail the challenges women and girls face with regard to inadequate facilities, infrastructure, products, education, and other means of support, we must acknowledge that experiences of menstruation are very context-specific and be careful not to overgeneralize from existing (small-scale) studies.

Third, there is a need to challenge the underlying assumptions of some interventions. As is often the case in global development, we must avoid the imposition of Western ideas, perceptions, or products without adequate regard for local contexts, such as the wholesale dismissal of menstrual cloth 
as a viable option. Such assumptions lose sight of the aim to enable empowered and informed decision-making, a move that can patronize menstruators. Because participation of the people concerned is key for something as personal as menstruation, many programs would benefit from devoting more time to understanding menstruators' priorities and preferences. Acknowledging the agency of women and girls and any menstruators requires that they must be able to decide how they want to cope on the days they menstruate, what activities and cultural practices they want to engage or avoid, and what materials they want to use to catch the flow (if at all).

Finally, there is a risk of rushing decisions and entrenching existing patterns of exclusion and marginalization. In our efforts to move menstruation from the margins to the center, do we leave some individuals behind? For instance, by framing menstrual health as an 'adolescent girls' issue,' do we ignore other women's health issues across the life course? Do we acknowledge women who do not menstruate as well as the needs and experiences of menstruators who do not identify as female? By focusing interventions on girls in schools, do we reinforce the marginalization of girls who are out of school? To respond to these challenges, future initiatives should consider how to most productively support, inform, and normalize the menstrual health of marginalized populations including people who are trans or gender nonconforming, live in poverty, experience homelessness, and/or are deprived of their liberty. We must tune into the experiences of people who are on the move or live with a disability, as well as others living in various conditions of precarity (see "Part I: Menstruation as Fundamental" of this Handbook). We need more emphasis on the lived realities and experiences of all menstruators to inform policy-making, programming, innovation, and data collection to ensure that no menstruators are excluded.

Policies and other instruments can be a powerful normalizer that recognize menstruation as requiring political attention, but such normalization and structural change can only be achieved when policy-making and programming include initiatives to challenge sociocultural norms and menstrual stigma. In order to bring about such structural change we need to think about menstruation in a comprehensive way and use it as a lens to identify and address injustices at the intersection of gender and a range of other identities. 
Open Access This chapter is licensed under the terms of the Creative Commons Attribution 4.0 International License (http://creativecommons.org/licenses/ by $/ 4.0 /)$, which permits use, sharing, adaptation, distribution and reproduction in any medium or format, as long as you give appropriate credit to the original author(s) and the source, provide a link to the Creative Commons license and indicate if changes were made.

The images or other third party material in this chapter are included in the chapter's Creative Commons license, unless indicated otherwise in a credit line to the material. If material is not included in the chapter's Creative Commons license and your intended use is not permitted by statutory regulation or exceeds the permitted use, you will need to obtain permission directly from the copyright holder. 